\title{
El editor de revistas académicas: institucionalidad y responsabilidad
}

Doi: https://doi.org/ 10.25100/hye.v16i54.10110

\author{
Alfonso Rubio \\ Universidad del Valle \\ Correo electrónico: alfonso.rubio@correounivalle.edu.co \\ ORCID: 0000-0002-5782-5092
}

Licenciado en Filología Hispánica y doctor en Sistemas de Información y Documentación por la Universidad de Zaragoza (España). Profesor del Departamento de Historia de la Universidad del Valle (Santiago de Cali, Colombia). Miembro del grupo Nación-Cultura-Memoria, dedica sus investigaciones a la historia de la cultura escrita. Ha sido editor de las obras Minúscula y plural: cultura escrita en Colombia (Medellín: La Carreta, 2016) y Miradas cruzadas: orden escrito, política y prensa en Colombia (Cali: Universidad Santiago de Cali, 2017). Algunas de sus publicaciones más recientes son La escritura del archivo: recurso simbólico y poder práctico en el Nuevo Reino de Granada (Cali: Universidad del Valle, 2014); Los escribanos de la Villa de Medellín, 1675-1819: la representación de un oficio en la escritura de su archivo (Medellín: Universidad de Antioquia, 2015); Pedro Herreros, 1890-1937. La hoja de vida de un poeta español en Buenos Aires (Logroño: Instituto de Estudios Riojanos, 2018); Memoria de un romance, La muerte a cuchillo (Madrid: CSIC, 2018); y, en coautoría con Juan David Murillo, Historia de la edición en Colombia, 1738-1851 (Bogotá: Instituto Caro y Cuervo, 2017).

Forma de citar este artículo: Rubio, Alfonso. "El editor de revistas académicas: institucionalidad y responsabilidad”. Historia y Espacio, vol. 16 n 54 (2020): 7-24. Doi.org/10.25100/hye.v16i54.10110.

Artículo Tipo 2: de reflexión 


\section{Presentación}

El pasado 15 de mayo de este presente año de 2020, Colciencias abrió una nueva convocatoria para la "indexación de revistas científicas colombianas especializadas" (Publindex, ${ }^{\circ} 875$ de 2020) ${ }^{1}$. Y de nuevo, año tras año, aparecen los trastornos y las inconformidades entre los editores de las revistas académicas colombianas. Se dio a conocer así un comunicado donde se informaba de una llamada "Propuesta de trabajo para la reforma de Publindex", elaboradapor una mesa "Mesa de Editores" que se creó en junio de este 2020 y en la que participaron, según dice el comunicado, cincuenta y dos editores. Entre ellos no estaba la representación de esta revista, Historia y Espacio, como no estaba la representación de muchas otras. La Mesa, se dice también en dicho comunicado, "ha venido avanzando por su cuenta" y ya cuenta con una "propuesta para modificar a fondo Publindex" que espera difundir prontamente.

Una vez más se pone en evidencia la falta de comunicación y de colaboración entre el conjunto de todas las revistas académicas del país. Claro que no es fácil trabajar colectivamente, pero las iniciativas personales que nacen en la institucionalidad pública deben darse a conocer antes de su desarrollo a todos a quienes compete el alcance de estas iniciativas. Estamos hablando de modificar la política pública de Publindex, con lo que, particularmente, por otro lado, estoy de acuerdo, no solo desde mi posición como editor de una revista académica, sino también desde mi posición laboral de profesor universitario.

Por un lado, hay un buen número de editores que, fieles a los dictados de Publindex, continúan acudiendo a cursos, jornadas o talleres sobre "métricas en las revistas académicas", o “cómo editar desde la Universidad”. Por otro, aunque resignados a seguir las políticas de Publindex porque podríamos "desaparecer del mapa”, estamos quienes ya desde hace un tiempo adoptamos una postura crítica encaminada a poder modificar los parámetros por los que se asigna una u otra categoría a una revista académica; encaminada, incluso, a poder anular la misma clasificación. Las divergencias, pues, son grandes entre los propios editores de las revistas y por ello no es fácil el consenso, de ahí que este deba partir de la institucionalidad de las propias universidades como tales.

El comunicado de esta "Mesa de Editores" puede ser un referente inicial válido para abrir una discusión donde se impliquen las instituciones competentes, primero para que pueda darse y, luego, para seguir avanzando

1 Convocatoria para indexación de revistas científicas colombianas especializadas. Publindex 2020. (https://minciencias.gov.co/convocatorias/fortalecimiento-capacidades-para-la-generacion conocimiento/convocatoria-para) 
en la mejora de las revistas sin que estas tengan que competir entre ellas. El objetivo de la Mesa, se dice en el comunicado, "apunta a que se cree un grupo técnico asesor de la Mesa Técnica Publindex con el propósito de producir una propuesta de reforma de Publindex que sea adecuada y conveniente para la ciencia, la comunicación científica y el país”.

El propósito general, se sigue diciendo, es avanzar en una "comunicación científica eficaz, actualmente distorsionada por el modelo Publindex”. Después de presentar algunas "problemáticas centrales" de este modelo, que realmente justifican su modificación y tienen que ver con cuartiles, índices citacionales o indicadores bibliométricos, se concluye hablando del régimen salarial de los docentes universitarios:

$<<$ El Decreto 1279 de 2002 establece el régimen salarial y prestacional de los docentes de las Universidades Estatales. El Artículo 10 se refiere a la productividad académica y es la base normativa de la clasificación de las revistas científicas colombianas, ya que establece las categorías de las mismas y los puntos que se pueden otorgar por la publicación de artículos.

Sería muy conveniente que se abordara la derogación o modificación del Artículo 10 del mencionado Decreto en el marco de un proceso de transformación del sistema de ciencia y tecnología, así como en la generación de una nueva visión de régimen salarial y prestacional de los docentes universitarios. En todo caso, debemos apuntar a que la calidad de las revistas científicas no sea la base central de los modelos para premiar económicamente la productividad académica $>>$.

La calidad, habría que precisar, a mi entender, vista exclusivamente desde los criterios establecidos por Publindex que, en sí, no miden la calidad objetiva del contenido de los textos. El comunicado acaba sugiriendolas siguientes medidas:

1. Suspender definitivamente el actual modelo Publindex a partir de septiembre de 2019, conservando la vigencia de los resultados de la convocatoria 830 de 2018, mientras se define un nuevo modelo.

2. Conformar un grupo técnico asesor de la Mesa Técnica Publindex, que rediseñe y proponga un nuevo modelo de Publindex, con amplia participación de los editores de las revistas científicas nacionales.

Estoy de acuerdo, desde mi particular opinión, como digo, en que nuestro esfuerzo académico, materializado en textos que acuden a las revistas para su conveniente difusión, puede ser medido desde parámetros muy distintos a los 
que actualmente nos juzgan, más atentos a la calidad real de textos. No podemos seguir sujetos a criterios estrictamente numéricos que nos impiden salir de las ataduras competenciales y nos obligan a contar con un aparataje técnico con el que muchas veces, al menos los departamentos de las universidades públicas, no podemos contar. Por ello y a partir de esta iniciativa, quiero dar a conocer aquí, pues nunca se ha difundido en un medio colombiano, el artículo que en el 2018 dio a conocer la Revista Amoxtli en su primer número. Lo hago con la idea de que en algo pueda contribuir a ese obligado debate que debe abrirse y no paralizarse, como ya ocurrió en ocasiones pasadas, hasta poder llevar a cabo la necesaria modificación de las políticas impuestas por Publindex. Permítanme, por último, una puntualización. La revista decidió publicar el artículo con el título cambiado de El editorderevistas académicas en Colombia: institucionalidad y actuales desafíos. El título original es el título con el que ahora se da a conocer aquí: El editor de revistas académicas: institucionalidad y responsabilidad. Una responsabilidad, por cierto, que es la que debe guiar la edición de nuestras revistas académicas y no el desafío por escalar hacia una categoría que, en numerosas ocasiones, no es reflejo de una justa calidad.

\section{Introducción}

La historia de cada una de las revistas académicas que sostienen la difusión investigativa de las universidades colombianas conlleva una serie de vicisitudes que lógicamente han implicado diferentes orientaciones programáticas y las ha convertido en un referente relevante no solo para el ámbito académico regional y local, sino también para el nacional y el latinoamericano. Las revistas universitarias siguen siendo una plataforma de intercambio de ideas y opiniones, donde se da cabida a una producción intelectual que desde múltiples maneras de abordar sus objetos de estudio, admiten diversas confluencias de enfoques investigativos. Las revistas por ello deben ser cuidadas para que se mantengan y no maltratadas o humilladas haciendo que desciendan de categoría o desaparezcan de la Plataforma de Publindex, el servicio de indexación de revistas académicas de Colciencias, el Departamento Administrativo de Ciencia, Tecnología e Innovación encargado de promover las políticas públicas para fomentar la ciencia en Colombia.

En consonancia con la apertura de la información científica ligada a tecnologías convenientes para su difusión, es el deseo de todas las revistas académicas proponer un entorno informático abierto, colaborativo y compartido que las envuelva para que diferentes escalas geográficas y diferentes 
sectores sociales puedan beneficiarse de sus contenidos. Esto implica un compromiso institucional de las universidades, que debe contar con un plan de recursos humanos, presupuestales y tecnológicos, dedicado exclusivamente a sus revistas y a su seguimiento. El aporte que desde muy diversos departamentos y facultades universitarias se hace a sus revistas es continuo desde hace muchos años y en numerosos casos no se ve correspondido con medidas de protección o el acompañamiento institucional, académico e investigativo, adecuado para mejorar la calidad de sus publicaciones y su difusión.

El acceso abierto es necesario contemplarlo en las políticas estatales y universitarias y es necesario el cumplimiento de sus compromisos con las exigencias previstas de evaluación y autoevaluación que beneficien la producción de un patrimonio intelectual concreto en beneficio de una comunidad académica más integral. Un acceso abierto dotado de herramientas prácticas y claras que facilite la lectura de aquellas publicaciones, producto de procesos docentes e investigativos que por su calidad lo ameriten. En este sentido, un nuevo ideal de apertura, nos dice Robert Darnton, a la manera de una República de las Letras ilustrada, está actualmente cambiando el mundo del conocimiento. Contamos con universidades, programas, metadatos y revistas de acceso abierto, pero lastimosamente esta tendencia tiene un lado oscuro y negativo, pues el acceso al conocimiento se está poco a poco obstaculizando y cerrando. La comercialización está en el origen de este cierre que va limitando al mismo tiempo el sentido democrático de la información bibliográfica (de "libros" y "revistas") y lo va dirigiendo hacia la privatización ${ }^{2}$.

Entre la institucionalidad colombiana que nos habla de categorizaciones que jerarquizan una posición académico-salarial de las revistas universitarias, cercana al mundo comercial de los llamados Índices Bibliográficos Citacionales, y la obligación laboral que se asume por el desempeño de unas funciones en un contexto continental determinado, no se hace nada fácil el desempeño del editor de revistas académicas para aplicar el principio desde el cual todo oficio debe desenvolverse: la responsabilidad. Está bien que se nos vea desde los rincones más recónditos del mundo virtual para poder satisfacer las necesidades de muy variados públicos, pero que se nos vea sin la mirada que solo ambiciona el ascenso competitivo y no la calidad de los escritos.

2 Darnton, Robert. Acceso abierto y otras reflexiones (Bogotá: Universidad de los Andes/Ediciones Uniandes, 2017), 1-17. 


\section{Deficiencias democráticas, déficit de relaciones institucionales}

Curiosamente, nuestras deficiencias democráticas explican cómo están estructuradas las instituciones públicas y cuál es el déficit de sus relaciones. En el año 2002, el Gobierno colombiano promulgó el Decreto 1279, que estable el régimen salarial de los docentes de las universidades estatales y asignó a Colciencias la función de diseñar y mantener el Sistema Nacional de Indexación y Homologación de Publicaciones Especializadas en Ciencia, Tecnología e Innovación (PUBLINDEX). Para su diseño y el establecimiento de sus políticas, pareciera lógica la colaboración de esta institución y el conjunto de las universidades públicas, los principales centros que editan y mantienen revistas científicas producto de su actividad investigativa. Pero esto no es así y este es el principal asunto del descontento generalizado que todavía existe entre los editores de las revistas universitarias y la comunidad académica en general.

Colciencias comienza legislando sin previas explicaciones de sus posicionamientos, sin una difusión pedagógica que haga comprender los nuevos lineamientos a que deben sujetarse los equipos editoriales de los distintos departamentos universitarios, sin colaboración alguna por parte de todas las instituciones implicadas en el fortalecimiento de un adecuado sistema, sin ni siquiera un mínimo de relación con quienes más se necesita, al menos eso experimentamos la mayoría de editores de revistas universitarias: silencio absoluto. No comprendemos el origen de los cambios sistémicos a los cuales Colciencias obliga a las revistas y parece que la potencial eficacia de la Plataforma de Publindex se construye sobre su silencio y el silencio de quienes la padecen. Escasas son las voces críticas, pero sí las hubo, recordemos, a las ambigüedades que surgieron a raíz de sus dos seguidas convocatorias de los años 2015 y 2016 para categorizar las revistas académicas. Estos fueron algunos de los comentarios personales que se hicieron y que relacionamos aquí porque creemos resumen el alto nivel de indignación que produjeron en los grupos de investigación y en el profesorado universitario en general. El texto se tituló "Colciencias: una nueva plataforma para un nuevo país", y decía así3:

$<<$ Sin, al parecer, remedio posible, Colciencias se sigue caracterizando por su falta de recursos investigativos y su desastroso hacer para estabilizar un adecuado sistema de revistas, con una permanente y paralizadora falta de planificación y con una "producción" de múltiples molestias y vaivenes

3 Rubio, Alfonso. Colciencias: ¿una nueva plataforma para un nuevo país?, Agencia de Noticias Univalle, Universidad del Valle, 2015. Disponible en: http://www.univalle.edu.co/ciencia-y-tecnologia/colciencias-nueva-plataforma. 
incomprensibles para los investigadores, a quienes más que como eso, se les trata como objetos investigados; que un año deben hacer una cosa, y al siguiente otra; que un año cuentan con un presupuesto de investigación y, al siguiente, normalmente disminuido, con otro; que un año se encuentran con una megabrillante plataforma, y al otro, se les cambia y se lessupercomplica, volviendo, eso sí, con el diseño de sus campos informativos, a los viejos esquemas que separabanlas denominadas"ciencias exactas", de las "ciencias humanas", porque cualquiera de los campos está hecho para eso, para la exactitud, para aumentar las diferencias, siempre desequilibrantes, entre los grupos; que un año, en fin,se ven sometidos a la aplicación deunos criterios de medición y, al siguiente, de otros; y así...hasta no se sabe cuándo, y así, como reza su eslogan, que es eslogan del gobierno nacional, "Todos por un nuevo país".

Todo, como si fuese la característica de una eterna im-potencia, parece funcionar a base de remiendos. Todas las políticas de Colciencias, desde que ha comenzado, parece ser que a modernizarsecon un mayor y más rígido control de los grupos de investigación del país, suponemos que benevolentemente necesario, lo único que hasta el momento han originado son trastornos, mentales ypolíticos, personales y grupales, nacionales y regionales.

Así, Colciencias, un aparato burocrático de Estado que debe coordinar el Sistema Nacional de Ciencia, Tecnología + Innovación y está conformado por los actores del conocimiento en la nación colombiana; en primer lugar, por la comunidad científica y académica, lo único que está legitimando essu mal hacer, porque a su existencia todavía no le hemos visto utilidad. Más queorientar, la política y los mandatos de Colciencias desorientan; más que estructurar, desestructura; más quenunca las numerosasquejas públicas por parte del profesorado investigadorestán justificadas.Si el control descontrolado (no planificado, sin plazos prudentes, con requisitos inoportunos) al que ahora se nos está sometiendo en esta nueva convocatoria de medición de grupos, ha de servir para algo, debe ser para dedicar definitivamente mayores presupuestos a la investigación, para organizar primero y articular después los centros nacionales y regionales oficiales con un funcionamiento real, para relacionar las necesidades investigativas regionales a una planificación nacional coherente, para dar cabida a parámetros que midan los esfuerzos desde otras lógicas no cuantitativas, para hacer caso, en definitiva, (Colciencias, en tanto organismo estatal, no se sitúa por encima de los grupos e investigadores, antes al contrario, se debe a ellos) auna rabieta que no deja de amplificarse por las 
diversas plataformas de Crítica, Trastorno + Indignación que últimamente están aflorando por la comunidad científica y académica del país >>.

Las sucesivas convocatorias de Colciencias siguen aumentando las disconformidades en el ámbito universitario, pero sí han hecho pensar en el sentido y las funciones actuales de sus revistas. El último modelo de Colciencias, según dice este mismo organismo, busca "incrementar el impacto de la producción científica colombiana, el número de revistas científicas nacionales incluidas en Índices Bibliográficos Citacionales (IBC) y la consolidación de revistas científicas nacionales en las áreas priorizadas en la política nacional de CTeI", con el fin de mejorar la "conexión entre calidad y estímulos al desarrollo científico y tecnológico del país” 4 . Tal vez, con estos cambios, el modelo valore con más rigurosidad la producción académica al asignar una más alta clasificación a las revistas que cuentan con mayores impactos en el panorama internacional. El modelo, por otro lado, está obligando a las revistas a ingresar en los IBC. Pero el modelo ha hecho que muchas revistas hayan desaparecido de la categorización de Publindex y muchas otras hayan descendido de categoría.

Publindex "excluye" y "desciende" al propio objeto que sostiene su razón de ser sin tomar remedios para ello, para mantener la dignidad que las revistas universitarias colombianas, que han sufrido de los nuevos criterios basados en las "bases de datos citacionales", han mantenido a lo largo de su historia y trayectoria académica. Si excluye su razón de ser, ¿de qué "conexión entre calidad y estímulos al desarrollo científico y tecnológico” está hablando? La calidad de las revistas universitarias no puede medirse exclusivamente con parámetros cuantitativos, algo obvio si reparamos en el significado propio de ambos sustantivos ("calidad"/"cantidad"), que frecuentemente oponemos entre sí y, en otras ocasiones, hacemos que el de "calidad" complemente al de "cantidad", pero no viceversa. El descenso y la desaparición de las revistas no se ve acompañado de estrategias por parte de las instituciones que quieren, dicen, "consolidarlas" y mejorar su impacto; por parte de las instituciones que pueden reorientar los criterios de medición, si es que estos, tal y como están ahora, hay que seguir manteniéndolos, pues las clasificaciones jerárquicas ligadas además a un salario profesoral, resultan ser una gran atadura de difícil resolución para mejorar la real calidad de los contenidos académicos. Antes al contrario, creemos que los empobrece.

4 Publindex (Página Web). Enlace: (http://publindex.Colciencias.gov.co:8084/publindex/ EnIbnPublindex/) 
Colombia, por otro lado, suele funcionar antes con la Ley que con la Educación o la Pedagogía y el Consenso para diseñarla, difundirla y ponerla en práctica. Pienso ahora en la Ley General de Archivos, la Ley 594 de 2000, que obliga a la organización documental de los archivos públicos y privados sin que la mayoría de los ciudadanos encargados de los mismos, cuando dicha ley comenzó a funcionar, supiera comprender los conceptos básicos de la archivística. Colciencias, como una característica propia de las instituciones públicas colombianas, legisla con el desconocimiento de las instituciones para las cuales lo hace, sin amplios consensos o suficiente representatividad y sin pedagogía previa antes de que sus nuevos planes programáticos se pongan en marcha.

Es posible que en un primer momento la Ley pueda concienciar ante los asuntos que propone regular, pero la Ley sin educación va siempre aparejada de un siempre maligno comportamiento: el temor. Frente al temor, la responsabilidad. Ejercer una profesión con temor solo conduce al trauma y a la perpetua sumisión que de manera incomprensible sigue lineamientos impuestos desde entes estatales superiores, como si solo de ellos dependiera, más que la solución, la salvación. La responsabilidad profesional y colaborativa, que parte de una pedagogía comprometida y transparente con la institucionalidad y con los ciudadanos para quienes deben dirigirse los servicios públicos, es pieza esencial en el desarrollo y mejora de la edición académica. Estamos hablando de profesiones o funciones como la de editor, íntimamente ligada, entre otras, a las ciencias de la comunicación social y, por tanto, la educación para comprender ciertos principios, ciertos objetivos y ciertas metodologías de unas nuevas prácticas editoriales, se hace completamente necesaria

Si se rompiera la cultura de la ocultación, que va ligada a la cultura de los grupúsculos y el clientelismo político-académico, tomaríamos conciencia de la magnitud del problema. Esto sería posible gracias, precisamente, y en parte, a la conexión de innumerables revistas que deben expresar su voz, primero personalmente; y, luego, unidos mediante una aserción colectiva. $\mathrm{Ni}$ una cosa ni la otra se da. Las iniciativas, sean los casos de las "conferencias", "jornadas", "seminarios", representan el lugar donde se comparten experiencias y Colciencias sigue actuando sin la opinión ni la colaboración de las propias revistas que se someten a sus dictados. Las iniciativas en este sentido, en cualquier caso, son prácticamente inexistentes en Colombia y este de la colaboración nacional sería un primer paso a partir del cual, solo a partir de él cabe la colaboración iberoamericana. Se necesita un plan de acción global en 
favor de las revistas científicas que atribuya un verdadero sentido estratégico en el mundo académico.

\section{La jerarquización y la categorización salarial}

Un reciente estudio del Reuters Institute for the Study of Journalism de la Universidad de Oxford, que se centra en el Reino Unido y podemos extrapolar al ámbito occidental, titulado I saw the new son Facebook, concluye para el año 2017 que más de la mitad de los ciudadanos se informan ya por medio de redes sociales. Más del 50\% de esa mitad no recuerda correctamente las fuentes de información, y estas así pierden autoridad y alisan las jerarquías. El asunto del aumento del número de lectores por redes sociales o soportes virtuales en general sí podemos ponerlo en relación con el mundo de las revistas académicas que ya, hoy en día, prácticamente la mayoría de ellas han dejado el soporte papel para centrarse en el soporte, la circulación y la lectura virtual. Pero pensamos que el asunto de la autoridad de las fuentes documentales y el de las jerarquías, siguen cobrando mayor relevancia en el entorno universitario que en cualquier otro ámbito.

En la esfera pública de lo rápido, casi lo inmediato de la comunicación, y con más información que nunca, que no mejor información, la responsabilidad de la autoría de los ensayos o artículos de investigación se deteriora cuando los encontramos hechos a manera de collage cuyas fuentes son engañosas al dar como leídas fuentes básicas, de autores principales, que en realidad están tomadas de citas secundarias o indirectas. Pareciera en el mundo universitario actual que basta con mirar pantallas virtuales para poder participar en la convocatoria de turno de una determinada revista. Por otro lado, las encuestas actuales hablan de que la lectura completa de libros es cada vez menor y en numerosas ocasiones esta se reduce a ciertos capítulos de los mismos. La lectura virtual potencia lo rápido y lo fragmentario y deja inconclusa la comprensión totalizadora de obras que hay que analizar en su totalidad para dar la rigurosidad apropiada a un trabajo investigativo o ensayístico.

El asunto de las jerarquías es más complejo y hay que relacionarlo con el cambio de modelo de la esfera pública: de esas personas privadas, reunidas como un público que delibera sobre las necesidades sociales y las articula con las políticas de Estado, como diría Habermas, hemos pasado a la privatización y comercialización de la difusión de nuestro conocimiento público a través de estándares que imponen empresas privadas al servicio de unas bases de datos 
cuya presencia en ellas marca una u otra categorización. Las revistas académicas se encuentran jerarquizadas.

Las sucesivas convocatorias lanzadas por Colciencias han ido incrementando los niveles de exigencia de los criterios que miden las revistas académicas (criterios de calidad científica y editorial, estabilidad o regularidad y visibilidad). La actualización de 2015, indexó 526 revistas en el nivel nacional. La convocatoria 768 de agosto de 2016 , con base en un rediseño del modelo de indexación que incorporó el criterio de la citación como parámetro que mide el impacto de las publicaciones y las clasifica según conocidos indicadores cienciométricos (Journal Citation Report (JCR), Scimago Journal Ranking (SJR) o el índice H5 propuesto por J.E. Hirsch); esta convocatoria, decimos, de acuerdo con estos indicadores y los criterios definidos por Colciencias en correspondencia con cada una de las cuatro categorías del modelo PUBLINDEX: A1, A2, B y C, hizo que unas desapareciesen del mapa de Publindex y otras descendiesen de categoría. No hay ninguna revista nacional en la categoría A1 y solo ocho de ellas se ubican en la categoría A2.

La falta de información previa por parte de Colciencias para poder y saber participar en los procesos de las convocatorias y, sobre todo, el propio modelo que emplea para categorizar, por sus ambigüedades y falta de correspondencia entre lo que se mide y la verdadera calidad de las revistas, por las inconsistencias en la definición del H5, han recibido innumerables críticas. Pero más allá de las críticas al modelo y de la continua incertidumbre que genera el sistema, hay que decir que según el Decreto 1279, la clasificación de cuatro jerarquías (A1, A2, B y C) se encuentra relacionada al salario de los profesores universitarios y mientras esto siga siendo así seguiremos sumergidos en la sumisión a unas redes privadas diseñadas como escaparate comercial para beneficiar los intereses de grandes tecnológicas ligadas a propuestas que miden el saber público.

Plataformas tecnológicas privadas que, sobre el aserto de McLuhan, hacen que el medio (y no el contenido) sea el salario. Los gobiernos, las instituciones como Colciencias, vigilantes de esta especie de catapulta académicocapitalista que representan las revistas científicas, deben ponerse de acuerdo con las universidades públicas para cambiar el modelo de ajuste salarial que jerarquiza, controla y vigila este tirano ecosistema digital. Desligar el salario de las categorizaciones descomplejizaría el trabajo en los departamentos, disminuiría los traumas que todavía sigue acarreando la participación en sucesivas convocatorias de Colciencias, acabaría con las presiones propias de la competitividad donde nos ha instalado el mundo externo empresarial, ajeno, 
en muchos casos, al mundo académico de las ciencias humanas; y no tendría por qué desmejorar la calidad de las revistas, que siempre debe asociarse a la responsabilidad académica de sus equipos editoriales. Seguramente seguirá habiendo nuevas reformas educativas universitarias y esperemos que ellas, que las nuevas leyes que nos rijan, cuenten con nuevos criterios evaluativos de la producción académica y que, al mismo tiempo, no nos eliminen la posibilidad de aumentar nuestro puntaje.

Los países, las universidades deben proteger sus revistas académicas ofreciendo buenas condiciones a la investigación y la difusión editorial de sus resultados. Se trata de trabajar por el bien común en proyectos cuya divulgación debe ser de dominio público. Lo que se hace en la Universidad pertenece a la humanidad y su difusión no es propiedad ni debe ser competencia de las grandes compañías tecnológicas.

No sabemos cómo ni desde qué momento los gobiernos, al lado del mundo empresarial privado, se pusieron a controlar la producción del conocimiento desde categorizaciones que nos han introducido en un sistema de macrodatos cerrado en sí mismo que impone lo igual y excluye lo distinto, pero este sistema, en realidad, no forma desde el sentido crítico y disminuye la calidad de sus resultados. Este sistema, como dice el filósofo surcoreano Byung-Chul Han, hace superfluo el pensamiento porque si todo es numerable, todo es igual5. Estamos en el mundo del pleno "dataísmo" donde los autores de producción académica ya no somos soberanos de nosotros mismos, sino resultado de una operación algorítmica que nos domina sin que lo percibamos, o lo percibamos viviendo en constante angustia de no hacer siempre todo lo que se puede, explotándonos a nosotros mismos pensando en que nos estamos realizando difundiendo ampliamente nuestro nombre en redes académicas. Pareciera que si no triunfamos es culpa nuestra y ya no hay contra quién dirigir la crítica. Es la alineación del profesorado universitario a través del mundo virtual con el cual no nos comunicamos, solo intercambiamos información: las relaciones se reemplazan por las conexiones y así solo no enlazamos con un sistema que únicamente reconoce lo igual. Cuanto más iguales sean las revistas y sus modos de operar, más aumenta la producción hasta el punto de llegar a leer que una revista que no llega a reunir más de 10 ó 15 artículos al año es índice de escasa

5 Han, Byung-Chul. Ahora uno se explota a sí mismo y cree que está realizándose, Diario El País, Edición impresa de la Ciudad de México, 7 de febrero de 2018. 
profesionalización6. La "empresa" no funcionaría si las revistas emplearan lógicas distintas. La universidad tiene clientes y solo produce trabajadores, no forma espiritualmente y el cambio pasa precisamente por un reajuste políticoeducativo de los responsables públicos.

\section{Las funciones del editor}

Las funciones del editor de revistas universitarias dependen de diversos factores que afectan al país donde ellas de emiten y a las universidades que por sus distintas regiones se distribuyen: alfabetización, recursos financieros, potenciales usuarios, idiosincrasia laboral y organizativa que complejizan el entorno de un desempeño que ya no es exclusivo de las funciones tradicionales del editor de revistas, sino también de las funciones de un nuevo campo laboral: la edición virtual. Esta exige un trabajo colectivo directamente ligado a la organización de equipos que necesitan de estabilidad laboral para poder adquirir destrezas y dar continuidad de calidad a los productos editoriales.

Pero no es fácil trabajar colectivamente y creo que esto se siente con intensidad en las universidades colombianas. La ambición, el orgullo personal, la desconfianza, las excesivas y complejas exigencias burocráticas, asuntos a veces potenciados por la comodidad del inmovilismo, la desorganización o los clientelismos y la politiquería institucional, hacen que las cargas editoriales sean un asunto molesto y lejano a intereses estrictamente académicos.

Los nuevos lenguajes virtuales hacen que las revistas tengan que adaptarse a las posibilidades del medio electrónico en correspondencia con las académicas. Esto exige contar con profesionales formados que preferiblemente hagan parte de la plantilla de la revista y para esto la financiación es asunto básico. Las malas prácticas prevalecen y la profesionalización de las revistas todavía es un objetivo pendiente, además de que todavía no se ha dado una reflexión crítica sobre las implicaciones del cambio de lo impreso a lo electrónico en el mundo académico de las revistas.

Las publicaciones se verían respaldadas si se propiciara un ambiente de apropiación y pertenencia del espacio físico y virtual donde se desenvuelven. Una revista debe contar con un crecimiento sostenible de sus recursos humanos que pueda hacer frente a cambios en su organización y su gestión, con un equipo en constante formación que pueda garantizar su futuro.

6 Rodríguez Yunta, Luis y Elea Giménez-Toledo. "Fusión, coedición o reestructuración de revistas científicas en humanidades y ciencias sociales". El profesional de la información 22:1 (Enero-Febrero, 2013), 36-45. Disponible en: http://eprints.rclis.org/18672/ 
Los profesores, como ya sabemos, no somos editores técnicos que debamos dedicarnos a las tareas materiales de la edición, a la gestión de metadatos, la identificación de bases de datos, al aprendizaje de lenguajes semánticos como el XML, o la difusión virtual, sino editores que debemos centrarnos en los contenidos, en la dirección de un equipo y en las decisiones que orienten la línea y la calidad de las revistas. Hay que reivindicar por ello las funciones tradicionales del editor responsable y esto nos lleva a un primer cuestionamiento, el del método de la evaluación por pares. La responsabilidad de un equipo editorial debería ser suficiente para dotar de calidad a una revista, para no poner en ridículo unos simples listados de miembros que forman un "comité científico" y otro "comité editorial" que se suelen confeccionar desde parámetros cuantitativos ligados al índice $\mathrm{H}$ de los mismos y no desde su utilidad, que muchas veces ni funcionan.

Al lado de la crítica a la evaluación por pares, por supuesto, el método de la indexación. Los criterios de calidad frecuentemente no se corresponden con los índices citacionales y frecuentemente desconocemos las causas (modas, amistades, trabajos colectivos) por las que un artículo intranscendente, en nuestra particular opinión, es más citado que otro que con certeza creemos es de mayor calidad. Este, entre otros, es uno de los motivos que nos lleva igualmente a desconfiar del sistema de indexación jerárquica como un método válido de ponderación de la calidad de las revistas.

Los editores más comprometidos saben que deben aumentar y diversificar considerablemente la cuota de autores y propuestas a difundir. Todavía se mantienen las rancias redes de amistad profesoral que repiten con frecuencia autorías y la idea, como editores, es la de ser verdaderos intermediarios entre autor y público académico. La programación de específicos dosieres, en este sentido, tal vez reduzcan las cuotas de citaciones, pero pueden, por otro lado, abrir nuevos caminos académicos no suficientemente tratados en determinados ámbitos académicos. En lugar de premiar las novedades, Publindex las castiga, pues la especificidad de ciertos dosieres tiende a la reducción, al menos a corto plazo, de su potencial citacional.

Ligadas a la responsabilidad editora, podemos citar, al menos para esta "región" del mundo, algunas medidas básicas que deben partir de una política institucional para mejorar el entorno de la edición académica y deben preceder a cualquier cuestionamiento técnico en el mundo de la edición académica: 
1. Asegurar el cumplimiento de las metas cuantitativas en cuanto a cobertura de educación básica y universitaria y subsidiar los niveles de posgrado mediante más y mejores ayudas al estudiante, para lo cual es necesario un fuerte incremento en la inversión educativa.

2. El fortalecimiento de la lectura y la escritura son las competencias que mejor garantizan un futuro educativo; competencias que indudablemente deben abrirse al consentimiento de la lectura virtual en relación a los nuevos soportes de presentación de los avances académicos.

3. Diseñar un programa conjunto que responda primero a las nuevas necesidades del sector universitario nacional y luego a las diversas aspiraciones continentales adecuadas a las exigencias de disponer de más y mejor educación para que enfrente el incierto futuro de viejos y nuevos empleos que ni siquiera podemos adivinar.

4. Garantizar una difusión y visualización inclusiva, equitativa y de calidad promoviendo oportunidades de publicación.

5. Es obvio que las competencias tecnológicas en la presentación virtual de los textos académicos han ocupado un valor relevante frente al impreso $\mathrm{y}$, por tanto, estas deben corresponderse con las acciones educativas que en todos los niveles educativos implementen medios tecnológicos de búsqueda y consulta de información.

6. Trabajar de otra manera, con el apoyo garantizado de las instituciones donde se inscriben las revistas y con prioridades y aliados expertos teniendo presente que no siempre "más" equivale a "mejor". Se trataría de articular institucionalmente las revistas de la universidad donde se editan, y crear un equipo sólido que se dedique al mantenimiento y mejoramiento de los procesos editoriales que cada vez son más exigentes técnicamente, tanto para su reconocimiento como para su visualización en determinados lenguajes virtuales y determinadas bases de datos.

7. Sin investigación no hay revistas. Frente a las voces académicas que hablan de una conveniencia a que las revistas estén afiliadas a "asociaciones profesionales", más que a las universidades, hay que decir que no se entienden muy bien sus apreciaciones, al menos en el ámbito colombiano, y parecen desconocer la situación de la producción de conocimiento académico que, como bien sabemos, la gran mayoría nace del ámbito universitario. Todavía, actualmente, el gran peso de divulgación de conocimiento recae en las universidades y mientras esto siga así, los esfuerzos habrá que dedicarlos a los medios divulgativos con que ellas cuentan. 
Sistemas de evaluación, tasas de citación, puntualidad de publicación, revisión por pares, diversidad regional, colaboración internacional en los comités científico y editorial, impacto global, interés coherente con la comunidad científica, todas estas son cuestiones que no es posible mejorar sin esfuerzos conjuntos que consigan audiencias más amplias, mejoren los servicios a autores y consultores y mejoren, en definitiva, la calidad de los artículos.

La historia de las revistas en Colombia, por último, no parece reflejar una acumulación de esfuerzos colectivos orientados a la inclusión, sino más bien al sálvese quien pueda. Los arreglos de Colciencias, los intereses corporativos de las empresas tecnológicas han suplantado el interés público y académico. La simulación o pruebas de medición en convocatorias públicas se han vuelto frecuentes y los trastornos que ellas ocasionan no se corresponden con la trayectoria personal de cada una de las revistas, que siempre han intentado aportar desde su particular y a veces modesto posicionamiento académico.

Publindex otorga categorías pero no formación para la calidad y el mantenimiento de las revistas que verdaderamente mejoren el trabajo investigativo. Se ensayan alternativas y soluciones pero se descarta el aprendizaje de la complejidad técnica que hace que los departamentos contraten a profesionales del mundo privado. No se dan incentivos para impulsar y llevar a cabo reformas en consonancia con la real idiosincrasia educativa de un país. Se quieren dar pasos transformadores y cambiantes decisivos en el sistema de revistas y, por tanto, en el sistema de educación universitaria, pero el andamiaje legal y operativo no ha sabido promocionarse por las inconsistencias y ambigüedades demostradas por Colciencias.

La cooperación institucional es la única vía posible de acrecentar la calidad dentro de una dimensión competitiva y esta no ha sido puesta en práctica con suficiente representación de las instituciones.

En definitiva, algunos asuntos son claves para poder considerar una futura reorientación política de Publindex:

1. Un cambio en los criterios de calidad que no estén ligados a los Índices Bibliográficos Citacionales.

2. La anulación de las jerarquías clasificatorias ligadas al puntaje salarial para cambiar los criterios evaluativos que relacionen la edición de artículos con el salario profesional. 
3. La consideración del editor de revistas que debe evaluar, corregir y reconocer el trabajo de la edición centrándose en los contenidos, en la dirección de un equipo y en las decisiones que orienten la línea y la calidad de su revista.

Entre los siglos XV al XVIII, los impresores y libreros europeos experimentaron, unas veces una, otra veces otra, las dos funciones del libro que, según Lucien Febvre y Henri-Jean Martin, este inspiró: la de ser mercancía y fermento ideológico o intelectual. El editor moderno se caracteriza por lo que Jean-Yves Mollier subraya, que sabe reunir bajo su batuta las dos funciones7. Los editores de revistas no debemos limitarnos exclusivamente a colgar en la red una convocatoria y esperar que se llene de distintas y jugosas especies de peces. Parecen ya perdidas las agallas del oficio y debemos recuperarlo buscando y reclutando nuevos autores capaces de conmocionar, si esto es posible hoy en día, el paisaje intelectual. La participación de los editores de revistas en los debates académicos e intelectuales del momento pueden ser un acicate para ello.

\section{Referencias}

Darnton, Robert.Acceso abierto y otras reflexiones (Bogotá: Universidad de los Andes/ Ediciones Uniandes, 2017), 1-17.

Han, Byung-Chul. Ahora uno se explota a sí mismo y cree que está realizándose, Diario El País, Edición impresa de la Ciudad de México, 7 de febrero de 2018.

Mollier, Jean-Yves. Del libro impreso al archivo digital. Fin de un ciclo para la edición mundial, Conferencia Inaugural, II Coloquio Argentino de Estudios sobre el Libro y la Edición,Córdoba 21 al 23 de septiembre de 2016.

Publindex (Página Web). Enlace: (http://publindex.Colciencias.gov.co:8084/publindex/EnIbnPublindex/)

Publindex. Convocatoria para indexación de revistas científicas colombianas especializadas. Publindex 2020. (https://minciencias.gov.co/convocatorias/fortalecimiento-capacidades-para-la-generacion conocimiento/convocatoria-para)

Rodríguez Yunta, Luis y Elea Giménez-Toledo. "Fusión, coedición o reestructuración de revistas científicas en humanidades y ciencias sociales”. El profesional de la información 22:1 (Enero-Febrero, 2013), 36-45. Disponible en: http://eprints. rclis.org/18672/

Rubio, Alfonso. Colciencias: ¿una nueva plataforma para un nuevo país?, Agencia de Noticias Univalle, Universidad del Valle, 2015. Disponible en: http://www.univalle.edu.co/ciencia-y-tecnologia/colciencias-nueva-plataforma.

7 Mollier, Jean-Yves. Del libro impreso al archivo digital. Fin de un ciclo para la edición mundial, Conferencia Inaugural, II Coloquio Argentino de Estudios sobre el Libro y la Edición,Córdoba 21 al 23 de septiembre de 2016. 


\section{Presentación del númerov15n54 de Historia y Espacio}

Las nodrizas afrodescendientes en el Nuevo Reino de Granada siglo XVII: valor cultural y científico, un artículo de María Cristina Navarrete, destaca el papel de las mujeres afrodescendientes que, actuando como amas de cría para la lactancia de los hijos recién nacidos en las pudientes, ejercían a la vez de mediadoras culturales.

Justicia y ley en el Nuevo Reino de Granada, periodo borbónico, de Isidro Vanegas, muestra cómo la Corona española estableció un conjunto institucional y normativo destinado a garantizar la convivencia. Cómo se concibió la ley en relación con el sentido de justicia, cuáles fueron sus rasgos y fundamentos y de qué fuente estos emanaban en la sociedad neogranadina, son los asuntos principales que el texto trata.

Roger Pita es el autor de Escollos y legados en la aplicación del armisticio de Trujillo en las provincias del sur de Colombia, 1820-1821. En el marco del Bicentenario de la Independencia de Colombia, este texto analiza el armisticio de Trujillo firmado en 1820 en relación con su implementación en las provincias del sur de Colombia, donde sentó las bases para la regularización de la guerra independentista.

Tras bambalinas: museografía y proyección de imaginarios nacionales en las Exposiciones Históricas (Madrid, 1892), es un artículo de Carmen Cecilia Muñozy Paula Revenga. En él se reflexiona sobre la proyección de imaginarios nacionales en los discursos museográficos desplegados por Portugal y Colombia en las Exposiciones Históricas que tuvieron lugar en el Madrid de 1892, en el marco de la celebración del cuarto centenario del descubrimiento de América.

Petróleo y ciudad en la Patagonia argentina, 1907-1940, de Javier Eduardo Serrano, analiza la relación entre industria petrolera y ciudad en la Patagonia argentina, desde el descubrimiento del primer yacimiento en 1907 hasta la década de los años cuarenta dentro de un proceso de estatización que aumentó la demografía y reconfiguró el espacio.

Indulgencias, coros y reconocimiento. La Revista de Misiones y las obras misionales pontificias en el mundo infiel, Putumayo, 1925-1930, de Giovanny Paolo Arteaga, se detiene en la Revista Misiones como fuente documental a partir de la cual examina la estrategia implementada por la Iglesia católica para financiar las Misiones en Colombia entre 1925 y 1930 y formar al clero indígena.

Willian Alfredo Chapman, Jesús Ángel Castro y Ángela Lucía Agudelo, en Trasformando el país a través de la higiene. Las cartillas técnicas y la revista Rin Rin en las bibliotecas aldeanas de Colombia 1936-1938, considera la función discursiva 
de higiene y progreso que desempeñaron en el ejercicio escolar las cartillas técnicas y la revista Rin Rin en el proceso de modernización de Colombia, bajo el gobierno del liberal Alfonso López Pumarejo.

La revista Micro (1940-1949) y la crítica cinematográfica y radiofónica en Colombia, es un texto de Andrés Villegas y Catalina Castrillón que se centra en otra revista, esta vez, la revista Micro, editada en Medellín entre 1940 y 1949. Desde la postura crítica que ella desplegaba, los autores plantean cómo se contribuyó a la profesionalización del cine y la radio nacionales.

William Bermeo, con Rasgosidentitarios del discurso de la movilización Minga de Resistencia Social y Comunitaria, identifica tanto los rasgos contextuales como los de contenido que caracterizan dicho discurso con una ideología antineoliberal.

Se incluyen, para finalizar, las reseñas escritas por Carlos Daniel Ortiz, Julián David Corredor y Víctor Manuel Gruel, dedicadas respectivamente a los libros de Jorge Augusto Gamboa Mendoza (Los Muiscas y su incorporación a la monarquía castellana en el siglo XVI: Nuevas lecturas desde la nueva historia de la Conquista), de Marilía Dalva Klaumman Cánovas (Santos e Imigração na Belle Époque. Os espanhóis - Cotidiano urbano, práticas associativas e militância política,1880-1922), y de Elizabeth Villa (Entre el vacío y la orfandad. Sociedady prácticas culturales en Tijuana, 1942-1968). 
